\title{
LA TRADIZIONE POLITICA DELLA RAGION DI STATO TRA UMANESIMO E MODERNITÀ
}

\author{
ALESSANDRO ARIENZO ${ }^{1}$
}

\begin{abstract}
L'espressione ragion di Stato nasce nel contesto della cultura politica del tardo Rinascimento con una pluralità di significati e usi che non è affatto riducibile - come spesso accade nel dibattito comune e pubblicistico - al solo ricorso alla forza, al segreto, o a strumenti eccezionali da parte del detentore del potere politico. Dal suo primo emergere nella cultura politica italiana di fine Cinquecento essa appare invece come parte determinante di un più complessivo processo di razionalizzazione politica che accompagna il consolidarsi degli stati territoriali moderni. In questo contributo vorrei illustrare i discorsi e le pratiche di governo che si affermano, tra fine XVI e inizio XVII secolo, in Italia ma non solo, nell'ambito della trattatistica sulla ragion di Stato. Per poi argomentare le ragioni per le quali essi contribuiscono a segnare tanto un percorso differente, anche se non necessariamente alternativo, al pensiero politico classico, quanto a consolidare gli assetti moderni della statualità, pur differenziandosi dal paradigma della sovranità.
\end{abstract}

PAROLE CHIAVE: ragion di Stato, conservazione politica, prudenza, machiavellismo, tacitismo.

ABSTRACT: The expression "reason of State" was born in the context of the political culture of the late Renaissance with a plurality of meanings and uses that cannot be reduced - as often happens - to the mere use of force, secrecy, or exceptional instruments by the holder of political power. From its first emergence in Italian political culture with the publication of Giovanni Botero's Della Ragion di Stato in 1589, it appeared as a relevant effort toward a more comprehensive process of political rationalization that accompanied the strengthening of territorial States. In this contribution I will briefly present the Italian and European early modern literatures on reason of State. I will also offer a brief sketch on the main historiographical approaches to reason of State in order to discuss how this tradition contributed to shape political modernity.

KEYWORDS: reason of State, political conservatism, prudence, machiavellism, tacitism.

\footnotetext{
${ }^{1}$ Alessandro Arienzo insegna Storia delle Dottrine Politiche all'Università degli Studi di Napoli "Federico II". Coordina i lavori del Centro Studi Ars Rosa su Ragion di Stato e democrazia (www.ragionidistato.it). Questo contributo sintetizza le linee di ricerca sulla ragion di Stato che, a partire dagli anni ' 90 del Novecento, e sotto la direzione di Gianfranco Borrelli, hanno caratterizzato l'Archivio della Ragion di Stato prima, e l'attuale centro studi Ars Rosa. E-mail: alessandro.arienzo@unina.it.
} 
Stato è un dominio fermo sopra i Popoli e Ragione di Stato è notizia di mezi atti a fondare, conservare, e ampliare un Dominio così fatto. Egli è vero, che se bene, assolutamente parlando, ella si stende alle tre parti sudette, nondimeno pare, che più strettamente abbracci la conservatione, che l'altre; e dell'altre più l'ampliatione, che la fondatione: imperò che la Ragione di Stato suppone il Prencipe e lo Stato, (quello quasi come artefice, questo come materia) che non suppone, anzi la fondatione affatto, l'ampliatione in parte precede. Ma l'arte del fondare, e dall'ampliare è l'istessa; perché i principi, ed i mezi sono della medesima natura. E se bene tutto ciò, che si fa per le sudette cagioni, si dice farsi per Ragione di Stato, nondimeno ciò si dice più di quelle cose, che non si possono ridurre a ragione ordinaria, e commune ${ }^{2}$.

\section{Premessa: la ragion di Stato tra machtpolitick e conservazione politica//}

Ragion di Stato è una espressione che appare nella cultura del tardo Rinascimento a significare quei principi politici, quelle tecniche e quei saperi necessari a garantire la conservazione dello Stato. Essa non è quindi riducibile né ai soli percorsi del machiavellismo e del tacitismo, né a quelli giuridico-politico della deroga, della prerogativa e della sovranità. La ragion di Stato esprime una differente istanza di razionalizzazione della politica che risponde al consolidarsi degli stati territoriali moderni e all'indebolirsi progressivo dei piccoli stati regionali.

È noto come nel celebre Die Idee der Staatsräson in der neuren Geschichte Friedrich Meineke abbia interpretato la ragion di Stato come machtpolitik, come unione di ethos e kratos. Essa esprime quella norma fondamentale dell'azione politica che indica all'uomo politico ciò che è necessario fare per conservare lo Stato "vigoroso e forte" ${ }^{3}$. Per lo storico e filosofo tedesco la ragion di Stato consisteva in un fenomeno essenzialmente moderno, dovuto alla nuova organizzazione territoriale della politica. Essa dava corpo alla personalità dello Stato e ne realizzava la sostanziale organicità culturale e vitalistica. Quella di Meinecke - per sua stessa ammissione - era la ragione dello Stato per come si era presentata tra Otto e Novecento, in parte derivazione dalle riletture organicistiche della tradizione machiavelliana e dei dibattiti Cinque e Seicenteschi sulla buona e sulla cattiva ragion di Stato. Tra gli anni '50 e '70 del Novecento, e in alternativa a questa lettura, prendono avvio una serie importante di studi storico-filologici che contribuivano a problematizzare questo approccio: si pensi ai contributi di Rodolfo De

\footnotetext{
${ }^{2}$ G. Botero, Della ragion di stato libri dieci di Giouanni Botero benese, in Torino, appresso Gio. Dominico Tarino, 1596, p.1.

${ }^{3}$ F. Meinecke, Die Idee der Staatsräson in der neuren Geschichte, München und Berlin, Druck und Verlag von R. Oldenbourg, 1925 (tr. it. L'idea della ragion di stato nella storia moderna, trad. it. D. Scolari, Sansoni, Firenze 1970).
} 
Mattei, di José A. Maravall, di Étienne Thuau, di Luigi Firpo e George L. Mosse E Carl J. Friedrich $^{4}$. E si pensi all'importante convegno di studi promosso da Roman Schnür e tenutosi a Tubinga nel $1974^{5} \mathrm{i}$ cui interventi hanno da un lato accolto la tesi della "modernità" della ragion di Stato, ma ne hanno offerto una caratterizzazione differente da quella meineckiana - perché basata sulla natura ordinaria della ragione della ratio status. Sono quegli gli anni in cui, non a caso, anche il filosofo francese Michel Foucault andava sviluppando una propria lettura della ragion di Stato ${ }^{6}$. Proprio sulla base di questi studi, un ulteriore momento di rinnovamento metodologico e storiografico si è avuto agli inizi degli anni Novanta del Novecento, quando, prima in Italia, quindi in molti altri Paesi europei, vi è stato una ripresa delle ricerche e l'acquisizione di importanti avanzamenti critici. Si vedano a tal proposito, per l'Italia, gli atti dei convegni Botero e la ragion di Stato del 1992, Aristotelismo politico e ragion di Stato del 1995 e La Ragion di Stato dopo Meinecke e Croce curati da Artemio Enzo Baldini, gli atti del convegno Ragion di Stato e ragioni dello Stato (secoli XV-XVII) del 1996 curato da Pierangelo Schiera, e gli atti dal titolo Prudenza civile, bene comune, guerra giusta del 1999 a cura di Gianfranco Borrelli ${ }^{7}$. Forse non a caso dopo il passaggio segnato dall' ' 89 , un ampio numero di studiosi italiani e europei ha raccolto l'eredità della tradizione di studi storico-filologico di studiosi come Rodolfo De Mattei, Luigi Firpo ed ha posto la necessità di allargare lo spettro di indagine delle scritture della ratio status all'intero ambito Europeo e non alla sola cultura "barocca" . Collocando, quindi, la ragion di Stato nel quadro del processo di composizione

${ }^{4}$ C.J. Friedrich, Constitutional Reason of State: the Survival of the Constitutional Order, Providence (R.I.), Brown University Press, 1957; G.L. Mosse, The Holy Pretence. A Study in Christianity and Reason of State from William Perkins to John Wintrop, Oxford, Basil, 1957; É. Thuau, Raison d'État et pensée politique à l'époque de Richelieu, Paris, Institut français d'Athènes, 1966; J.A. Maravall, Estado moderno y mentalidad social. Siglos XV a XVII, 2 voll., Madrid, Revista de Occidente, 1972; L. Firpo, La ragion di Stato. Appunti e testi, Torino, Bottega d'Erasmo, 1976; R. De Mattei, Il problema della 'ragion di Stato' nell'età della Controriforma, Milano-Napoli, Ricciardi, 1979.

${ }^{5}$ R. Schnür (hrsg.), Staatsräson. Studien zur geschichte eines politischen Begriffs, Referate des internationalen Kolloquiums über die geschichtliche Rolle des Begriffs der Staatsräson, Tübingen von 2. bis 5. April 1974, Berlin, Verlag Duncker \& Humblot, 1975.

${ }^{6}$ In particolare, in: Il faut défendre la société: cours au Collège de France, 1976, éd. M. Bertani, A. Fontana, Paris 1997; e Sécurité, territoire, population. Cours au Collège de France, 1977-1978, sous la dir. de F. Ewald et A. Fontana, par M. Senellart, Paris, Seuil-Gallimard 2004 (tr. it. Sicurezza, territorio, popolazione. Corso al Collège de France 1977-1978, Milano, Feltrinelli, 2005).

${ }^{7}$ A. E. Baldini (a cura di), Aristotelismo politico e ragion di Stato, Atti del Convegno internazionale di Torino, 11-13 febbraio 1993, Firenze, Olschki, 1995; P. Schiera (a cura di), Ragion di Stato e ragioni dello Stato (secoli $X V-X V I I)$, Atti del Convegno dell'Istituto per gli studi filosofici e dell'Istituto storico italo-germanico di Trento, 9-10 luglio 1990, Napoli, L'Officina Tipografica, 1996; A.E. Baldini (a cura di), La ragion di Stato dopo Meinecke e Croce: dibattito su recenti pubblicazioni, Atti del Seminario internazionale di Torino, 21-22 ottobre 1994, Genova, Name Press, 1999; G. Borrelli (a cura di), Prudenza civile, bene comune, guerra giusta. Percorsi della ragion di Stato tra Seicento e Settecento, Atti del Convegno internazionale, 22-24 maggio 1996, Napoli, ARS, 1999.

${ }^{8}$ Per una complessiva ricostruzione dei momenti più importanti della storiografia sulla ragion di Stato, cfr. il saggio di G. Borrelli, Gli studi di Ragion di Stato negli ultimi due decenni del ventesimo secolo: motivazioni e considerazioni critiche, "Politics. Rivista Italiana di Studi Politici", 4, 2, 2015, pp.1-13. Mi permetto di segnalare 
dello scenario moderno della politica e andando oltre i confini della machtpolitick presenti nella lettura del Meinecke.

\section{Botero e la ragione di Stato}

Proprio la definizione di Stato e di ragion di Stato citate in esergo ci permettono di cogliere il senso di questo conflitto tra le interpretazioni intorno al senso e alla modernità della ratio status. I passi sono quelli celebri che Giovanni Botero offre nell'edizione del 1596 nel suo trattato. Nella prima edizione veneziana dello stesso testo (1589) il capitolo recita più succintamente che lo Stato è: «un dominio fermo sopra i popoli; e Ragione di Stato è notizia di mezi atti a fondare, conservare, e ampliare un Dominio così fatto. Egli è vero, che se bene, assolutamente parlando, ella si stende alle tre parti sudette, nondimeno pare, che più strettamente abbracci la conservazione, che l'altre; e dell'altre più l'ampliazione, che la fondazione» ${ }^{9}$.

Proprio queste due differenti definizioni offerte dal benese nelle due edizioni dell'opera ci permettono di introdurre modalità diverse di pensare e interpretare la ratio status, e il senso della trattatistica italiana Cinque e Seicentesca. Innanzitutto, Botero definisce lo Stato come un "fermo dominio" su di un popolo e la ragione di Stato come quell'insieme di conoscenze (la notizia) dei mezzi necessari non solo a fondare e ampliare, ma anche a conservare un tale dominio. Nell'edizione del 1596 risalta la distinzione, che non si trova esplicitata nell'edizione del 1589, tra una ragion di Stato intesa come una ragione "straordinaria" di governo, e una «ragione ordinaria e commune» che - della ratio status - ne costituisce la parte più rilevante. A questa distinzione se ne affianca anche una seconda, che separa le ragioni dell'istituzione e dell'ampliamento di un dominio da quelle della sua conservazione. In entrambe le formulazioni boteriane, appare comunque evidente come il mantenimento del dominio resta il nucleo essenziale della ragion di Stato.

Definendo lo Stato come «dominio fermo sopra i popoli» e la Ragione di stato come «notitia di mezi atti a fondare, conservare et ampliare un dominio», Botero offriva le coordinate essenziali nonché il modello fondativo di quello che si potrebbe descrivere come nuova

solo alcuni importanti studi di area sudamericana: R. Romano, Razão de Estado e Outros Estados da Razão, São Paulo, Editoria Pespectiva, 2014; R. Jacarandá, Os pressupostos filosóficos do maquiavelismo e o surgimento da via moderna em política, "Veritas", 61, 1, 2016, pp.130-153.

${ }^{9}$ G. Botero, Della ragion di stato libri dieci di Giouanni Botero benese, in Venetia, appresso i Gioliti, 1589, p.1. $\mathrm{Su}$ Botero e le vicende editoriali della Ragion di Stato si veda l'introduzione di C. Continisio a G. Botero, $L a$ Ragion di Stato, Roma, Donzelli, 2009 e l'introduzione di R. Descendre a G. Botero, Della Ragion di Stato, Torino, Einaudi, 2016. 
"ragione di governo". La notitia cui il benese fa riferimento sono infatti i "saperi" e l'insieme delle conoscenze necessarie al retto governo del territorio e dei suoi abitanti: il fine principale è in definitiva conservare e far sviluppare la popolazione su cui si esercita il proprio dominio. In tal senso, all'inizio della sua trattazione, di fronte al problema di «qual sia opera maggiore, l'aggrandire, o '1 conservare uno Stato», Botero chiarisce che:

Senza dubbio, che maggior opera si è il conservare, perche le cose umane vanno quasi naturalmente hora mancando, hora crescendo, à guisa della Luna, à cui sono soggette: onde il tenerle ferme, quando sono cresciute, sostenerle in maniera tale, che non scemino, e non precipitino, è impresa d'un valor singolare e quasi soprahumano [...]. S'acquista con forza, si conserva con sapientia; e la forza è commune à molti; la sapienza è di pochi ${ }^{10}$.

Proprio in ragione di questa finalità conservativa, e della logica strumentale che la sottende, cambia profondamente anche lo statuto della prudentia e della sapientia, ora intese non più soltanto come virtù morali, ma come strumenti pratici di intervento politico, attraverso i quali il principe può misurarsi con le instabili passioni degli uomini, che rendono l'esercizio del governo sempre esposto al rischio dei romori e dei sollevamenti ${ }^{11}$. Se intende conservare il suo dominio il principe deve, secondo Botero, «conoscere la natura, gl'ingegni e l'inclinationi de' sudditi» ${ }^{12}$, ovvero

la notitia di tutte quelle cose che spettano alla cognitione degli affetti e de' costumi (che si dichiarano copiosamente da' filosofi morali) o alle maniere de' governi (che si esplicano da' politici), perché la morale dà la cognitione delle passioni comuni a tutti, la politica insegna a temperare o secondare queste passioni e gli effetti che ne seguitano ne' sudditi, con le regole del ben governare ${ }^{13}$.

Nell'opera di Botero l'arte di governare si articola in una pluralità di campi di intervento che abbracciano sfere molteplici dell'esistenza: certamente gli ambiti della produzione e della circolazione dei beni, dell'organizzazione degli eserciti e delle finanze, la diplomazia e la "geografia politica", l'urbanistica e l'educazione. Una attenzione particolare, tuttavia, è per tutti

\footnotetext{
${ }^{10}$ G. Botero, Della ragion di stato, 1589, pp.5-6.

${ }^{11}$ Cfr. V. Dini, La prudenza da virtù a regola di comportamento: tra ricerca del fondamento ed osservazione empirica, in V. Dini, G. Stabile, Saggezza e prudenza. Studi per la ricostruzione di un'antropologia nella prima età moderna, Napoli, Liguori Editore, 1983, pp.13-125, p.77. Ancora sui mutamenti della semantica politica e morale della prudenza cfr.: D. Taranto, Le virtù della politica. Civismo e prudenza tra Machiavelli e gli Antichi, Napoli, Bibliopolis, 2003; V. Dini, Il governo della prudenza. Virtù dei privati e disciplina dei custodi, Milano, FrancoAngeli, 2000 e M. Viroli, Dalla politica alla ragion di Stato. La scienza del governo tra XIII e XVII secolo, Roma, Donzelli, 1993.

${ }^{12}$ G. Botero, Della ragion di stato, 1589 , p.53.

${ }^{13}$ Ivi, p.48.
} 
quei settori della vita sociale che coinvolgono in maniera diretta gli interessi e le passioni private dei sudditi. Proprio per questo nella ragion di Stato boteriana è centrale la categoria di interesse, poiché, come lo stesso benese dichiara nelle Aggiunte alla Ragion di Stato, «ragion di Stato è poco altro, che ragion di interesse» ${ }^{14}$ : ossia, il governo prudente persegue una organizzazione della vita politica in cui devono avere pieno riconoscimento anche le ragioni degli interessi particolari. In un significativo passo dei Capitani dedicato al duca Carlo Emanuele, Botero afferma che: «invero tra tutte le opere di prudenza civile non ve n'è alcuna più commendabile, che quella con la quale gl'interessi privati co' pubblici si congiungono» ${ }^{15}$. La ragione di Stato non è quindi semplicemente una ragione politica tesa alla sopravvivenza di un dominio o al mantenimento, da parte del principe, del suo potere, ma una più complessiva ragione di calcolo dell'interesse dello Stato. Un interesse che risulta dalla razionalizzazione e composizione "politica" degli interessi particolari che lo abitano ${ }^{16}$. In questo senso, si può forse affermare che nella ragion di Stato la prudenza politica diviene un'opera di comunicazione e di mediazione tra corpi sociali diversi. Non è allora un caso che, nel solco della lunga tradizione aristotelica, il governante deve sapersi affidare ai più "conservatori” tra i propri sudditi, quelli appartenenti ai ceti "mezzani”.

La proposta boteriana configura allora una ragione dello Stato che, articolata in una serie di tecniche e di saperi specifici, mira a razionalizzare al massimo le potenzialità del comando esercitato dal principe o dal magistrato. A dispetto di quanto una rappresentazione corrente lascerebbe pensare, la ragion di Stato nasce con l'intento di coinvolgere il numero più ampio di individui e corpi sociali nella produzione "dinamica" di poteri, finalizzati alla conservazione dell'ordine esistente. La ragion di Stato in Botero è in sostanza una ragione politica che si ordina intorno alla necessità di costruire uno specifico rapporto di comando/obbedienza che - a differenza dal modello hobbesiano - non è centrato sui processi astratti dell'autorizzazione politica e della rappresentanza, ma al cui centro vi si trova il concreto governo dell'insieme di relazioni tra le parti del corpo politico; un governo che deve rendere possibile la conservazione dinamica di un dominio fermo.

\footnotetext{
${ }^{14}$ G. Botero, Aggiunte alla Ragion di Stato, Venetia, presso Gio. Battista Ciotti, 1598, p.34.

${ }^{15}$ G. Botero, I Capitani, Torino, appresso G. Tarino, 1607, p.228.

${ }^{16}$ Sugli interessi di stato, in rapporto alla ragion di Stato, vedi: D. Taranto, Studi sulla protostoria del concetto di interesse. Da Commynes a Nicole (1524-1675), Napoli, Liguori, 1992 e il saggio dal titolo Ragion di Stato e ragione di interesse, in P. Schiera (a cura di), Ragion di Stato e ragioni dello Stato, op. cit., pp.189-245.
} 


\section{La letteratura italiana sulla ragion di Stato}

È ormai noto che da un punto di vista strettamente filologico, l'atto di nascita della ragion di stato è di difficile individuazione. Ci sono studiosi per i quali la categoria della ragion di stato coinciderebbe con una sorta di arcano immutabile costitutivo dell'esercizio del potere politico, per altri sarebbe invece essenzialmente fondata su un recupero di temi giuridici medievali ${ }^{17}$. Tuttavia, la maggior parte degli studiosi oggi concorda sul carattere circoscritto e congiunturale della nascita della locuzione, se non del concetto. Se il suo primo utilizzo si trova in due luoghi cinquecenteschi (nel Dialogo del reggimento di Firenze, Francesco Guicciardini fa riferimento all'esistenza di una «ragione e uso degli Stati» ${ }^{18}$ e nell'Orazione a Carlo $V^{19}$, Giovanni Della Casa parla di «ragion degli Stati»), è certamente a Giovanni Botero che si deve la sua prima formalizzazione paradigmatica.

L'opera di Botero darà allora avvio a una vera e propria tradizione scritturale, un corpus relativamente omogeneo di testi che attraversa la letteratura politica italiana almeno fino alla metà del Seicento, suscitando attenzione e commento ${ }^{20}$. Questa tradizione accoglie e discute la distinzione boteriana tra una ragion di Stato come ragione commune e ordinaria finalizzata alla conservazione e una ragione diversa, straordinaria, connessa alla sicurezza e all'accrescimento dello Stato. Ma esprime anche l'esigenza di tenere separate e distinguere tra una retta e una corrotta ragione di Stato. A partire da Apolinnare de' Calderini che nei Discorsi sopra la Ragione di Stato di Giovanni Botero (1597) scriverà di ammirare e riverire il benese: «il quale col suo veramente alto ingegno ha formato una ragion di stato, la più giusta, la più onesta la più degna e meritevole di nessun altro, e ha dimostrato con vive ed efficaci ragioni che così deve procedere il Prencipe per mantenimento della giustizia e dello stato suo...» ${ }^{21}$. Calderini coglie in modo particolare proprio il discorso boteriano sull'interesse che guida gli animi degli uomini;

\footnotetext{
${ }^{17}$ Cfr. G. Post, Ratio Publicae Utilitatis, Ratio Status and “Reason of State”, 1100-1300 in Id., Studies in Medieval Legal Thought: Public Law and the State 1100-1322, Princeton, PUP, 1964, pp.241-309. Anche M. Senellart, Machiavélisme et raison d'État, Paris, PUF, 1989.

${ }^{18}$ F. Guicciardini, Del reggimento di Firenze, (1521-1527) in Opere, a cura di Roberto Palmarocchi, Milano, Rizzoli, 1941-1942, vol. II, p.666.

${ }^{19}$ L'Orazione a Carlo V si trova in Rime, et prose, Vinegia, presso Niccolò Bevilacqua, 1558; le citazioni sono alle pp.69 e 62. Per la ricostruzione e l'interpretazione di questo documento vedi R. De Mattei, I1 problema della “Ragion di Stato” nell'età della Controriforma, Milano-Napoli, Ricciardi, 1979, pp.10-12.

${ }^{20}$ Su questi percorsi rinvio a: G. Borrelli, Ragion di Stato e Leviatano. Conservazione e scambio alle origini della modernità politica, Bologna, Il Mulino, 1993; e Id., Ragion di Stato. L'arte italiana della prudenza politica, in Ragion di Stato. L'arte italiana della prudenza politica, catalogo della mostra bibliografica, Napoli, Palazzo Serra di Cassano 4-30 luglio 1994, Napoli, IISF, 1994.

${ }^{21}$ Apollinare de' Calderini, Discorsi sopra la Ragione di Stato di Giovanni Botero, Milano, P. M. Locarno, 1597, cit. p.61.
} 
un principio che renderebbe possibile l'accordo tra le virtù cristiane, l'onestà e il giusto col profitto de' Prencipi:

ricorderò quel detto di Botero in quel suo bellissimo libro, ch'egli intitulò della Ragion di Stato, degno veramente di essere scolpito in lettere d'oro nell'animo di coloro che negotiano con Prencipi, che l'interesse de Prencipi è quello che vince ogni partito, poi che dove si giuoca l'interesse loro, essi bene spesso non hanno legge, se non quella sola che li dà l'interesse stesso ${ }^{22}$.

Molto diverso sarà invece l'atteggiamento da parte di Fabio Albergati, espressione di quella parte cattolica ancora incerta rispetto alla compiuta proposta boteriana. Per Albergati ragion di Stato resta sinonimo di machiavellismo, effetto ulteriore dell'empietà dello scrittore fiorentino che ha disgiunto la morale dalla politica. Nell'opera La republica regia (1627), egli ribadirà che contro la ragione e l'interesse di Stato rimangono valide le ragioni naturali e morali per le quali: «'1 sapere operare per ragione di Stato assolutamente, cioè corrispondente a tutti gli stati, e repubbliche, è opera del legislatore universale, o diciamo dell'architettonico, o vogliamo dire del prudente civile che, conoscendo tutte le forme de' governi, sa conforme a tutti operare ${ }^{23}$. Se di ragion di Stato si vuole parlare, essa deve rimanere strettamente congiunta alla prudenza civile a garanzia del solido legame di onesto e utile, di virtù e impegno civile. Proprio su questo tema interviene anche Federico Bonaventura con lo scritto - pubblicato postumo - Della Ragion di Stato et della prudenza politica (1623); anch'egli in forma polemica nei confronti della sistemazione teorica realizzata da Giovanni Botero. Bonaventura vuole dimostrare che ragion di Stato appartiene ai campi della virtù morale e della prudenza civile e che essa si lega alle due principali funzioni della prudenza: quella legislatrice e quella giudiziale. Se rettamente intesa, la ragion di Stato, allora, «muta, e altera sempre, e corregge secondo il bisogno»; poiché essa consiste in una virtù che «non contraviene alla legge, non la restringe, come alcuni falsamente hanno stimato; ma talhora l'interpreta, ove ne sia bisogno e la dilata; onde non si può dire che sia contraria alla legge, e ragion ordinaria, ma sopra di essa» ${ }^{24}$. A sua volta nei Ragguagli di Parnaso $(1612)^{25}$, Traiano Boccalini svolge una critica radicale alle teorie e alle pratiche della ragion di Stato, dando anche conto di quanto diffusa fosse proprio l'espressione di ragion di Stato nel linguaggio quotidiano. Da Tacito a Machiavelli, dal Della

\footnotetext{
22 Ivi, p.23.

${ }^{23}$ F. Albergati, La republica regia, Bologna, Vittorio Benacci, 1627, cit. p. 579

${ }^{24}$ F. Bonaventura, Della Ragion di Stato et della prudenza politica libri quattro, Urbino, Alessandro Corvini, 1623, p.31.

${ }^{25}$ T. Boccalini, Ragguagli di Parnaso. Centuria prima, Venezia, Pietro Farri, 1612.
} 
Casa a Botero: a tutti costoro, sostenitori dell'arte del governo prudenziale, Boccalini dedica pagine irriverenti e ironicamente critiche.

Nonostante le critiche del Boccalini, gran parte dei teorici della ragion di Stato fondano le loro riflessioni proprio sulla compiuta autonomizzazione della prudentia politica: una nuova virtù che opera proprio sulla base della codificazione di quelle tecniche che rendono possibile al soggetto politico gli adattamenti conservativi necessari a garantire il controllo e il governo dinamico delle relazioni di potere. Tentativi importanti di codificazione dei dispositivi tecnici prudenziali, sui quali non possiamo ora soffermarci, sono nelle opere di autori come Pietro Andrea Canonieri; Gabriele Zinano; Fabio Frezza, Toquato Accetto ${ }^{26}$.

Più attente allo spostamento teorico operato dall'opera boteriana sono invece le osservazioni di Girolamo Frachetta secondo cui la ragion di Stato consiste innanzitutto nell'insieme dei dispositivi di governo attraverso i quali il principe garantisce la disciplina dei popoli a partire dal suo stesso disciplinarsi: «La Ragion di Stato è una pedìa o perizia, o disciplina che vogliam dire, nascente parte dagli insegnamenti altrui, parte dal senso e parte dell'isperimento delle cose del mondo, per la quale altri governa le bisogna sue o di chi sia, secondo che r'chiede il commodo di colui di cui sono» ${ }^{27}$. Secondo Frachetta l'azione politica del principe deve tendere a realizzare un rapporto positivo di comando ed obbedienza: di qui la ricerca di consenso da parte del popolo. Tutti i prìncipi «hanno bisogno del consentimento del popolo, o immediato, o espresso, o mediato, o tacito» ${ }^{28} \mathrm{e}$, a questo fine, produrre disciplina è dare ordine allo Stato. Ancora una volta, emerge l'intreccio tra le ragioni del governo e quelle dell'interesse, ma a queste "due ragioni" Frachetta ne affianca almeno una terza:

é da sapere che ci sono tre termini assai usitati appo gli scrittori e appo i favellatori politici. Il primo termine è Ragion di Stato, il secondo è Interesse di Stato, il terzo è Ragione di guerra. Significa, adunque, Ragion di Stato, nel modo che comunemente suol prendersi, una diritta regola colla quale si governano tutte le cose, secondo che richiede l'utile di colui cui appartengono. Interesse di Stato vuol dire il commodo di chi si sia, concernente il mantenimento delle cose che quel tale possiede e

\footnotetext{
${ }^{26}$ T. Accetto, Della dissimulatione honesta, Napoli, Egidio Longo, 1641. A. Canonieri, Quaestiones ac discursus in duos primos libros Annalium C. Cornelii Taciti, Romae, ap. Barthol. Zanettum, 1609; Id., Dell'introduzione alla Politica, alla Ragion di Stato e alla pratica del buon governo, Anversa, appr. Iochino Trognesio, 1614; Id., Il perfetto corteggiano, et dell'Uffizio del Prencipe verso il Cortegiano, Romae, per Bartol. Zannetti, 1609. G. Zinano, Della Ragione de gli Stati libri XII, Venezia, appr. Gio. Guerigli, 1626; Id., Il Segretario, Venezia, appr. Gio. Guerigli, 1625; Id., Il Consigliere, ove si mostra con qual'arte e accortezza debba procedere in tutt' $i$ consigli per ben pubblico d'ogni Stato, Venezia, appr. Gio. Guerigli, 1625. F. Frezza, Discorsi politici et morali, Napoli, T. Longo, 1617.

${ }^{27}$ G. Frachetta, L'idea del libro de' governi di Stato et di Guerra, Venezia, app. Damian Zenaro, 1592. Pure importanti sono: Il seminario de' governi di Stato, et di guerra, Venezia, per Evangelista Deuchino, 1617; e Il Prencipe, Roma, Niccolò Muzio, 1597.

${ }^{28}$ G. Frachetta, Il seminario de' governi di Stato, et di guerra, VII, p.30.
} 
l'ampliamento di esse. Ragione di guerra significa una diritta regola di ben governare le faccende militari ${ }^{29}$.

Al cuore della distinzione boteriana tra una ragion di Stato ordinaria e comune e una straordinaria vi è la riflessione di Scipione Ammirato la cui posizione può essere sintetizzata nella nota espressione per cui «ragion di stato altro non essere che contraventione di ragione ordinaria per rispetto di publico beneficio, overo per rispetto di magiore e più universal ragione $»^{30}$. Ammirato parla della ragion di Stato come di un particolare jus politico, che deroga all'ordinario jus publicum, e in cui la conservazione politica appare come il mantenimento del dominio attraverso l'esaltazione della centralità del potere politico. Nell'esercizio di una peculiare ragione di Stato da intendersi come esercizio di una ragione "universale" dello Stato, il principe si colloca al vertice della gerarchia dei poteri esercitando un governo che, proprio perché universale e straordinario, permette di evitare il ricorso alla violenza o di tenere il popolo in una condizione di continuo timore.

L’istanza di conservazione e stabilità, nel tempo, del dominio - declinazione specifica della fermezza boteriana - si trova anche nel discorso conservativo di Giovanni Antonio Palazzo, la cui riflessione prende avvio, nella Ragion vera di Stato (1604), proprio dalla descrizione delle caratteristiche di ciò che può chiamarsi uno Stato: «Stato è una identità e pace temporale delle cose; cioè un esser sempre la stessa essenza e una costanza delle cose nell'operare...; Stato è lo stesso dominio e potestà de' Prencipi, che si dice stato dall'intenzione di quelli, ch'è sempre di farlo stabile e fermo» ${ }^{31}$. La ragion di Stato è esercizio della prudentia politica necessaria a porre rimedio a tutto ciò che turba la quiete e la perfetione dello Stato. Intenzione particolare di Palazzo è in particolare definire la vera e buona ragion di Stato, a fronte di quella falsa e cattiva, perché è solo la retta ragion di Stato a operare come freno alle passioni naturali e a garantire la costanza, cioè la permanenza e la regolarità, nei comportamenti dei sudditi. Certamente, non scompare affatto il principio della "deroga", poiché è inevitabile che il: «Prencipe può per ragioni di stato trasgredire l'humane leggi, non essendo a quelle soggetto, come anco perché sogliono le leggi umane col tempo scoprirsi irragionevoli, essendo il più delle volte l'attioni humane tutte imperfette ${ }^{32}$. Il tema delle tecniche indispensabili alla stabilità del governo è pure al centro anche dell'opera di Ottavio Sammarco Delle mutationi de'

\footnotetext{
${ }^{29}$ G. Frachetta, L'idea del libro de' governi di Stato et di Guerra, pp.37-38

${ }^{30}$ S. Ammirato, Discorsi sopra Cornelio Tacito, Fiorenza, F. Giunti, 1594, p. 231.

${ }^{31}$ G.A. Palazzo, Discorsi del governo e della ragion vera di Stato, Venezia, presso Gio. Antonio e Giacomo de' Franceschi, 1606, pp.12-13 e 14 (pr. ed. Del governo e della ragion vera di Stato, Napoli, per G.B. Sottile, 1604). ${ }^{32}$ Ivi, p.378.
} 
regni $(1628)^{33}$ in cui alla prudenza politica è attribuita la funzione di garantire continuità al dominio evitando novità improvvise.

Diverso è il tema che intorno alla ragion di Stato si presenta nell'opera di Lodovico Zuccolo, un autore che tenta di gettare un ponte tra l'arti del governo secondo prudenza politica e la tenuta delle leggi civili. Nella voce dedicata alla ragion di Stato nelle Considerazioni politiche e morali $(1621)^{34}$, egli sostiene che le pratiche di ragion di Stato costituiscono solo una parte della funzione di governo; la politica persegue complessivamente la felicità di chi obbedisce e di chi comanda e deve garantire la partecipazione del popolo a sostegno dell'autorità politica; non a caso, la migliore forma istituzionale di governo viene considerata la repubblica, benché nel quadro della politica contemporanea non possa, secondo Zuccolo, che assumere come necessaria la forma di governo del Regno: proprio questa scelta comporta di accogliere le tecniche della prudenza politica, seppur col preciso intento di combattere comunque la tirannide. Zuccolo ritiene infatti che all'autorità politica del re spetti innanzitutto di accordare il piano delle leggi naturali e morali con quello delle leggi civili tramandate dalla consuetudine, e che l'ampio potere di deroga che è affidato al principe sia unicamente finalizzato a guadagnarsi il favore dei sudditi. Diversi ancora sono i contributi di Scipione Chiaramonti e di Lodovico Settala. Nel Della ragion di Stato (1635) il primo discute l'esistenza di una molteplicità di ragioni di Stato, le quali - certamente con argomentazioni diversificate possono tutte egualmente pretendere ad autonomia di pratiche applicative. L'autore cerca di offrire una descrizione della ragion di Stato ponendo in relazione i due campi di giustizia e prudenza: «la buona ragion di Stato, per l'abito presa, a due si riduce: 1'uno è la giustizia universale, l'altra è la prudenza politica, delle quali quella ha il diritto, questa l'utile dello Stato per oggetto» ${ }^{35}$.

Per contro, nel suo Della ragion di Stato $(1627)^{36}$, Lodovico Settala organizza la propria riflessione nel riferimento diretto al quinto libro della Politica aristotelica, assumendo a criterio espositivo delle modalità di attuazione della ragion di Stato le tre forme di governo rette e le altre tre oblique. Dalle sue argomentazioni emerge come egli intenda sostenere che tutte le forme di Stato conosciute, nel loro esistere "di fatto", sono essenzialmente ordinate secondo l'utile di chi governa. In tal senso, se la ragion di Stato di Settala si propone di perseguire la

\footnotetext{
${ }^{33}$ O. Sammarco, Delle mutationi de’ regni, Napoli, L. Scoriggio, 1628.

${ }^{34}$ L. Zuccolo, Considerationi Politiche, e Morali sopra cento oracoli di Illustri Personaggi antichi, Venezia, M. Ginami, 1621.

${ }^{35}$ S. Chiaramonti, Della Ragione di Stato, Fiorenza, stamp. di Pietro Nesti, 1635, p.437.

${ }^{36}$ L. Settala, Della Ragion di Stato libri sette, Milano, G.B. Bidelli, 1627.
} 
finalità morale del sommo bene - subordinando l'interesse particolare del principe a quello dello Stato - nei fatti però accoglie il procedere autonomo del governo a partire dagli interessi del detentore del potere. Si potrebbe allora argomentare che nella sua opera, la distinzione tra una buona e una cattiva ragione di Stato entra in un "corto-circuito" che rivela la preminenza di una dimensione moralmente neutra e strumentale dell'agire politico prudenziale.

In ultimo, l'opera di Virgilio Malvezzi ${ }^{37}$, segna forse con la maggiore chiarezza l'esaurirsi - nelle sue forme rigidamente prudenziali - della letteratura della ragion di Stato. Egli infatti denuncia l'inefficacia di queste pratiche politiche sulla base di una più ampia critica alla forma generale della politica a lui contemporanea: «i fatti de’ Principi hanno ogn'altra faccia che la vera», mentre il sostegno principale della politica viene riposto nell'esercizio della forza ( $R, p p$. 7 e 11$)^{38}$. Malvezzi osserva innanzitutto il procedere incontrollato e senza misura delle tecniche politiche dissimulative e simulative che, a lungo andare, diventano elemento di degrado del governo. Il tempo di intervento della prudenza politica non è più capace di provvedere ad una conservazione di tipo dinamico, risultando piuttosto in un irrigidimento dell'orizzonte della politica. Malvezzi mostra quindi il convincimento che questo modello "forte", ma ormai rigido, di praticare il comando politico non consente più di produrre una disciplina attiva e durevole da parte dei sudditi.

\section{La ragion di Stato nella cultura politica europea}

La riflessione italiana intorno alla ragion di Stato ha caratteristiche particolari, che prendono corpo nella cultura umanistico-rinascimentale italiana e che maturano nell'orizzonte "barocco" e controriformistico. Tuttavia, essa incrocia e si innerva in una più generale riflessione europea sulle arti del governo e sulle nuove ragioni degli stati. Certamente, non sono ancora indagate a sufficienza l'influenza della proposta boteriana e delle scritture italiane della ragion di Stato nella riflessione politica europea dei decenni successivi; fatte forse le debite eccezioni per quanto attiene ai percorsi esplicitamente riconducibili al machiavellismo e al tacitismo ${ }^{39}$. E se il contesto italiano ci consegna un corpus relativamente omogeneo di scritti che da Botero giunge forse al suo termine con la pubblicazione del testo di Chiaramonti nel

\footnotetext{
${ }^{37}$ Le sue principali opere sono: Discorsi sopra Cornelio Tacito, presso Marco Ginammi, Venetia, 1622; Il Romulo, presso Clemente Ferroni, Bologna, 1629; Il Tarquinio Superbo, presso Clemente Ferroni, Bologna, 1632; Davide Perseguitato, per Giacomo Monti, Bologna, 1634, Il ritratto del privato politico christiano, per Filippo Ghisolfi, Milano, 1635; L'Alcibiade e Il Coriolano, entrambi presso Dozza, Bologna, 1648.

${ }^{38}$ V. Malvezzi, Il Romulo, op. cit., p.7.

${ }^{39} \mathrm{Si}$ veda il volume a cura di G. Borrelli, Prudenza civile, bene comune, guerra giusta, op. cit., 1999. Per quanto concerne i percorsi del "machiavellismo" si veda G. Procacci, Machiavelli nella cultura europea dell'età moderna, Roma-Bari, Laterza, 1995.
} 
1632, ricostruire il tragitto europeo del lemma e del concetto di "ragion di Stato" resta un impegno ancora tutto da perseguire. Una tale impresa è resa certamente difficile da una serie di questioni teoriche che sono, a nostro parere, ancora da sciogliere appieno. La prima, e forse più rilevante, è data proprio dal ruolo e dalla collocazione del testo boteriano nel quadro più ampio di una trasformazione nei saperi sulle arti del governo e sullo Stato che, secondo linee di sviluppo assolutamente differenziate, precedono lo scritto del benese. Se il gesuita torinese scrive nel contesto della cosiddetta "contro-Riforma cattolica", egli certamente raccoglie e rielabora un nucleo di temi che derivano dalla trattatistica politica umanistica. Pertanto, si potrebbe legittimamente individuare in Botero un punto di svolta nella trattatistica sulle arti del governo, ma si potrebbe altrettanto legittimamente argomentare che il suo testo si colloca in una sostanziale continuità con il passato umanistico-rinascimentale. In secondo luogo, ai fini della ricostruzione dell'influenza che la ragion di Stato esercita sulla letteratura politica europea tra la fine del Cinquecento e per almeno tutto il Seicento, si pone l'urgenza di indagare il contributo diretto della letteratura italiana all'emergere di un modo nuovo di pensare e praticare le arti del governo e dello Stato, ma anche di ricostruire una più complessiva "temperie culturale" che dalla letteratura umanistica, attraverso le letture di Machiavelli e di classici come Tacito, Livio, Cicerone e Aristotele, giunge a Botero, ma anche a Lipsio e ai tanti percorsi del machiavellismo, del tacitismo, della ratio status primo moderna. Se non la lettera della ragion di Stato, questo percorso potrebbe piuttosto far emergere un orizzonte di temi e di problemi accomunati dall'istanza di pensare lo Stato a partire dalle necessità del suo governo. Ed in effetti, se non sono molte le tracce che portano "Botero in Europa", non è invece difficile tracciare, anche per grandi linee, i percorsi di una più ampia trattatistica politica sulle arti del governo dello Stato a diverso titolo riconducibile all'orizzonte teorico della conservazione politica. Si pensi, ad esempio, allo sviluppo di discorsi e pratiche di ragion di Stato che in Francia è ampiamente presente: nel II capitolo della seconda parte del suo Testament politique Richelieu ne individua i criteri ispiratori nello scrivere che «la raison doit être la règle et la conduite d'un État» (II, II: 8). Il cardinale fa esplicito riferimento alla necessità di applicare la prudenza politica secondo il principio tutto pratico della "dolcezza" (douceur), col fine di spingere all'obbedienza gli uomini attraverso la persuasione e il riconoscimento ragionevole degli interessi delle parti. In tal senso, la principale norma del governo secondo prudenza è: «négocier sans cesse, ouvertement ou secrètement, en tout lieux» (II,VI: 24) ${ }^{40}$. Allo stesso

\footnotetext{
${ }^{40}$ A. du Plessis, cardinal duc de Richelieu, Testament Politique, Paris, 1688.
} 
modo, le annotazioni di Mazzarino nel Breviarium politicorum ${ }^{41}$ avevano segnalato la necessità di far riferimento alle arti prudenziali proprie di una comunicazione "sdoppiata", tra visibile e invisibile, nel governo dello Stato. Altre modalità di espressione di una nuova ragione di governo e che sono sicuramente prossime ai dispositivi di tipo prudenziale sono quelle suggerite in Francia negli scritti di Montchrestien, per il quale governo politico deve prendersi cura della vita complessiva dei cittadini, del loro lavoro e del loro benessere. Nella amministrazione delle relazioni tra governati e governanti è necessario saper favorire la rete "degli scambi", perché se gli individui sono liberi di operare per il proprio arricchimento, il principe può più facilmente acquisire consenso ed offrire una forma gerarchicamente ordinata ai poteri. Nell'area francese, come termine di mediazione e di sviluppo "interno" delle pratiche di sovranità, la politica espressa dall'arte della conservazione tende decisamente a diventare police: arte della conduzione della popolazione nel suo complesso, al fine di conservare il potere e favorire, al contempo, il "benessere" - e non il bene - comune. In questo caso, il monarca è impegnato a favorire la produzione di saperi tecnici, amministrativi ed economici, adeguati. L'emergere dell'economia politica nel seno delle scritture dall'oeconomica rinascimentale, nel contesto dei nuovi saperi di governo rappresenta bene l'allargamento delle funzioni di diretto interesse politico - e quindi della natura - dello Stato $^{42}$. Per quanto concerne i paesi dell'area germanica - come ha invece ricostruito Michael Stolleis, a partire dalla fine della guerra dei Trent'anni, ed in particolare dall'opera di Conring del 1640, prende avvio un'ampia letteratura dedicata ai temi della ragion di Stato, in riferimento diretto in questo caso agli sviluppi propri dell'aristotelismo politico ${ }^{43}$. L'attenzione è allora posta a codificare quei dispositivi di governo che rendono possibile l'amministrazione e la gestione governamentale del popolo: di qui la proposizione di una copiosa produzione di saperi che si condensa intorno alla scienza di Polizia (Polizeiwissenschaft) e al Cameralismo ${ }^{44}$. Scienza della Police, Polizeiwissenschaft, policy, governo camerale: sono tutte modalità differenti di istruire un'autorità di comando, che pur

${ }^{41}$ G. Mazarino, Breviarium politicorum secundum rubicas mazarinicas, Coloniae Agrippinae, Typis Iannis Sellica, 1684.

${ }^{42}$ Cfr. P. Sebastianelli, Homines oeconomici. Per una storia delle arti di governo in età moderna, Roma, Aracne, 2017 e I. Hont, Jealousy of trade. International Competition and the Nation-state in Historical Perspective, Cambridge Mass, Belknap Press, 2005.

${ }^{43}$ Cfr. M. Stolleis, Staat und Staaträson in der frühen Neuzeit. Studien zur Geschichte des öffentlichen Rechts, Suhrkamp, Franckfurt 1990 (tr. it a cura di G. Borrelli, Stato e ragion di stato nella prima età moderna, Bologna, il Mulino, 1998); R. Schito, Alla ricerca della sovranità. Fonti e percorsi nella Germania del XVII secolo, Milano, Carocci, 2011 e M. Scattola, Politica architectonica. L'aristotelismo politico nel dibattito politico tedesco della prima età moderna, "Res Publica. Revista de Historia de las Ideas Políticas", 19, 1, 2016, pp.15-33.

${ }^{44}$ Tra tutti si veda il volume di P. Schiera, Il cameratismo e l'assolutismo tedesco. Dall'arte di governo alla scienza dello Stato, Milano, Giuffrè, 1968; cfr. anche H. Dreitzel, Protestantischer Aristotelismus und absolutistischer Staat, Wiesbaden, 1970. 
affondando le loro radici nell'aristotelismo politico, operano secondo un differente modello teorico che tenta di costruire un più diretto legame tra l'orizzonte giuspubblicistico della sovranità - di matrice bodiniana - e le nuove arti camerali del governo.

Altrettanto interessante è il caso dell'Inghilterra, dove all'uso limitato nella prima metà del Seicento della locuzione di ragion di Stato alle condizioni di esercizio della prerogativa assoluta da parte del sovrano, viene via via sostituendosi, a partire dalla metà del secolo, nei trattati ma anche nei discorsi propriamente politici, una elaborazione che affianca temi e prospettive prudenziali ai percorsi della sovranità parlamentare o anche di più specifici percorsi di tipo repubblicano. Nonostante la mole di studi sui diversi aspetti della cultura politica inglese tra Cinque e Seicento, e sulle sue molteplici connessioni con le tradizioni teoriche continentali, restano ancora ad oggi pochi gli studi sistematici sull'influenza della letteratura italiana della ragion di Stato in Inghilterra, fatta eccezione per quella parte riconducibile piuttosto alle traiettorie del machiavellismo e del tacitismo. I lavori espressamente dedicati a questo argomento sono a tutt'oggi relativamente pochi, e variamente distribuiti nell'arco di oltre secolo. Dopo gli importati studi di Francis Wormuth dedicati alla prerogativa assoluta e di George Mosse, dobbiamo attendere la più complessiva ripresa degli studi sulla ragion di Stato che prende avvio nel corso degli anni ' 70 del Novecento per vedere ripreso il tema della sua declinazione inglese nella prima modernità ${ }^{45}$. Per giungere, oggi, ai più recenti studi di Quentin Skinner, Richard Tuck, Geoff Baldwin, Istvan Hont, Thomas Poole, Jamie Trace e Joanne Paul. Questa letteratura mette bene in risalto quanto difficile sia un raffronto tra la cultura politica inglese della fine del Cinquecento e del Seicento con la letteratura italiana della Ragion di stato che da Botero prende avvio. Eppure, la questione, oltre che il vocabolario, della ragion di Stato appaiono correntemente nel linguaggio politico dell'epoca: Laws of State, Prerogative, Policy, Statecraft, Judgment, Mystery of State, oltre ai più consueti Interest of State, public Good, common Weal, Discretion, State Necessity, Ratio imperandi, arcana imperii, salus populi. A voler sintetizzare - semplificando forse troppo - sono almeno due le traiettorie di ciò che, a diverso titolo, ed almeno per tutta la prima metà del XVII secolo, possiamo indicare come English Reason of State: una relativa ai particolari sviluppi della cultura machiavelliana di cui l'epoca elisabettiana fu coltivatrice, che nelle categorie di policy e statecraft trova il suo centro teorico; l'altra che fa riferimento ad un percorso distinto che getta le sue radici in una differente

\footnotetext{
${ }^{45}$ Cfr. F. D. Wormuth, The Royal Prerogative. 1603-1649, New York, Cornell University Press, 1939; G.L. Mosse, The Struggle for Soveraignty in England, East Lansing, Michigan State College Press, 1950; G.L. Mosse, The Holy Pretence, op.cit., 1957; D.S. Berkowitz, Reason of State in England and the Petition of Right, 1603-1629, in Staatsräson, a cura di R. Schnur, Berlin, Duncker \& Humblot, 1975, pp.165-212.
} 
tradizione "giuridico-politica", che registra gli sviluppi inglesi delle idee di sovranità e di absolute Prerogative. È importante allora mettere in risalto come nella seconda metà del secolo, dall'incrocio di questi percorsi, attraverso le vicissitudini connesse alla fase rivoluzionaria e parlamentare, si sia strutturata una modalità più complessa di tematizzare la ragion di Stato. Essa appare come 1'articolazione di una ragione dello stato, ossia di una "scienza della politica" capace di produrre governo, conservazione e amministrazione di una realtà politica e istituzionale non più riducibile alla funzione centrale, al "corpo" del monarca. Parte, forse, di un più complessivo processo di "spersonalizzazione" dello Stato che non caratterizza affatto in via esclusiva la ragion di Stato. Nella traiettoria della ratio status inglese della seconda metà del secolo, al cuore di questo percorso di affermazione di un sapere dello Stato e di un sapere intorno alla ratio dello stato, due autori sono fondamentali, forse non a caso entrambi espressione dello schieramento repubblicano e parlamentare. In ambito parlamentare è innanzitutto Henry Parker ad argomentare una organizzazione del potere politico che si fondi sia sull'esercizio ordinario fissato dai termini della Common Law, sia su pratiche straordinarie di deroga di cui pure il Parlamento deve prendere responsabilità in modo da consentire sicurezza e benessere per la popolazione ${ }^{46}$. James Harrington, da parte sua, nel System of Politics ${ }^{47}$, definirà il compito della ragion di Stato come pratica corrente dell'amministrazione degli interessi dello Stato al fine di garantire il giusto equilibrio tra produzione/distribuzione della ricchezza e forma specifica del governo. La finalità esplicita è quella della conservazione dell'organizzazione statuale: amministrare significa dover ammettere sia l'esercizio del potere politico ordinario sia l'applicazione di dispositivi prudenziali straordinari. Per tornare, invece, in un ambito monarchico, e nel quadro della restaurazione Stuart, George Savile, marchese di Halifax, medierà tra queste diverse istanze nella sua concezione del trimmer, figura di uomo politico capace di coniugare una serie di prerogative di comando con gli sviluppi ordinari delle leggi civili e le competenze dell'amministrazione e della gestione istituzionale. Analoghe considerazioni andrebbero fatte per quanto attiene ai percorsi propri di ciò che potremmo definire come una "ragion di Stato repubblicana" che prende avvio in Olanda e nelle Province Unite nel corso del Seicento, evidentemente nel contatto diretto con l'esperienza britannica e nel contrasto con quella spagnola oppure a quelli di una specifica "ragione di Stato della

\footnotetext{
${ }^{46}$ Cfr. M. Mendle, Henry Parker and the English Civil War. The political thought of the public's "privado", Cambridge, CUP, 1995.

${ }^{47}$ In The Political Works of James Harrington, ed. With an Introduction by J.G.A. Pocock, Cambridge, CUP, pp.833-854.
} 
Chiesa"48. Una simile rassegna resta comunque parziale: importanti studi sono stati dedicati anche a ricostruire gli svolgimenti autonomi dei machiavellismi e della ratio status nelle aree dell'est Europa su cui è necessario ancora approfondire le ricerche.

\section{Conclusioni: la ragion di stato tra umanesimo e modernità}

Nel cinquantennio che segue alla pubblicazione del testo di Botero, il dibattito italiano sulla ragion di Stato mette in risalto l'emergere di un programma di discorsi e di dispositivi tecnici originale. La trattatistica della Ragion di Stato si presenta in un numero considerevole di interventi e mostra approcci differenti alla conservazione politica e alle arti del governo dello Stato: non è affatto scorretto affermare che esistono, in tal senso, diverse ragioni di Stato. Nonostante questa ricchezza, a metà del Seicento cessa in Italia il dibattito strutturato e fondativo sulla ragion di Stato. Tuttavia, è grazie al confronto tra discorsi e pratiche di governo differenti nei diversi contesti europei, e in particolare nella penisola italiana, che a partire dalla seconda metà del Cinquecento, si è andata consolidando una parte importante della cultura della conservazione politica. In consapevole discontinuità con il pensiero di Machiavelli, in questi testi si tentava di individuare quelle modalità attraverso le quali introdurre pratiche di governo capaci di garantire la conservazione degli assetti di potere esistenti ${ }^{49}$.

In tal senso, si può sostenere che nella storia europea - a partire dalla fine del Cinquecento e fino ad oggi - alcune istanze di concentrazione e di razionalizzazione del comando politico finiscono per riassumere nell'espressione di "ragion di Stato" una serie di percorsi teorici che contribuiscono a pensare e produrre ordine politico e sicurezza sociale. Lungo questo percorso teorico, riflettere sulle "ragioni" dello Stato significa porre il problema della effettiva esistenza e della conservazione dello Stato, piuttosto che dei processi della sua legittimazione giuridica o d'autorizzazione ${ }^{50}$. Peraltro, proprio gli studi più recenti sulla ragion di Stato hanno fatto emergere, oltre al riferimento importante, ma riduttivo all'eccezione e alla deroga, alcuni elementi paradigmatici di qualcosa di molto vicino a una "scienza dello Stato". Una scienza che accoglie il carattere storico-congiunturale dello Stato e ne indica alcuni elementi “paradigmatici”. Il primo è senz'altro l'emergere del territorio come spazio concreto

\footnotetext{
${ }^{48}$ Sull'esperienze "olandesi” si vedano gli studi di Hans Blom e Jan Waszink; sulla ragion di "Chiesa" il recente volume a cura di G. Borrelli, L. Coccoli (a cura di), Ragion di Stato e Ragioni della Chiesa, Napoli, Bibliopolis, 2019.

${ }^{49}$ Cfr. G. Borrelli, Machiavelli e la ragion di Stato: segnare con cura le differenze, in Stato, Nazione, cittadinanza. Studi di pensiero politico in onore di Leonardo La Puma, a cura di R. Bufano, Lecce, Milella, 2016, pp. 51-64. Cfr. anche Id., Machiavelli, ragion di Stato, polizia cristiana. Genealogie I, Napoli, Cronopio, 2017.

${ }^{50}$ Cfr. A. Arienzo, G. Borrelli, Emergenze democratiche. Ragion di stato, Governance, Gouvernementalité, Napoli, Giannini Editore, 2011.
} 
dell'esercizio del potere ${ }^{51}$. Il secondo è lo sviluppo di un sapere politico pratico e una scienza dell'amministrazione ${ }^{52}$. Il terzo è l'affermazione della politica come istanza autonoma, dotata di una razionalità conservativa propria distinta dal dominio della morale, del diritto e della religione $^{53}$. Un ultimo elemento di caratterizzazione dei trattati sulla ragion di Stato, che si troverà sviluppato pienamente nel neo-stoicismo della prima metà del Seicento ${ }^{54}$, è la definizione di quelle disposizioni individuali che rendono possibile l'obbedienza da parte del popolo attraverso pratiche di "autogoverno" e di disciplina/disciplinamento di governanti e governati ${ }^{55}$.

In tal senso, la trattatistica della ragion di Stato ci permette di dare una più articolata rappresentazione della "modernità politica", delle sue origini e dei suoi esiti. E non lungo un consueto e - forse semplicistico - continuismo tra il discorso "storico" del machiavellismo, della ragion di Stato e il contemporaneo realismo politico ${ }^{56}$. Al contrario, si tratta di intendere diversamente la genesi della modernità politica nel suo razionalizzare lo Stato. Come ha osservato il filosofo francese Michel Foucault, se è vero che lo Stato come realtà storica nasce ben prima della sua "ragione", è forse solo con la riflessione sulla ragion di Stato che quest'ultimo «entra effettivamente nella pratica riflessiva degli uomini» ${ }^{57}$. Con la ragion di Stato, in sostanza, lo Stato moderno «ha iniziato a essere progettato, programmato e sviluppato all'interno di una pratica di riflessione» ${ }^{58}$, ovvero a diventare oggetto di una conoscenza attenta, precisa e meticolosa, in grado di offrire tecniche e strategie per l'esercizio di effettive e concrete pratiche di governo.

I discorsi e le pratiche di governo che si affermano, tra fine XVI e inizio XVII secolo, in Italia ma non solo, nell'ambito della trattatistica sulla ragion di Stato contribuiscono allora a segnare un percorso differente, anche se non necessariamente alternativo, sia al pensiero politico classico quanto a quelli della statualità moderna. Distinta dal discorso della sovranità,

\footnotetext{
${ }^{51}$ Cfr. M. Stolleis, Staat und Staaträson, op.cit.; anche M. Senellart, Les arts de gouverner, op. cit.

${ }^{52}$ Cfr. C. Lazzeri, D. Reynié, La raison d'état: politique et rationalité, Paris, PUF, 1992.

${ }^{53}$ Per una trattazione sul tema della "conservazione politica" come paradigma conservativo: G. Borrelli, Non far novità. Alle radici della cultura italiana della conservazione politica, Bibliopolis, Napoli 2000.

${ }^{54}$ Tra i molti studi, cfr. almeno R. Tuck, Philosophy and government, 1572-1651, Cambridge, CUP, 1993.

${ }^{55}$ Cfr. G. Oestreich, Filosofia e costituzione dello Stato moderno, Napoli, Bibliopolis, 1989; ma anche i molteplici lavori di P. Schiara raccolti in Specchi della politica. Disciplina, melancolia, socialità nell'Occidente moderno, Bologna, Il Mulino, 1999

${ }^{56}$ Sul realismo politico: P.P. Portinaro, Il realismo politico, Roma-Bari, Laterza, 1999; A. Campi, S. De Luca (a cura di), Il realismo politico. Figure, concetti, prospettive di ricerca, Soveria Mannelli, Rubbettino, 2014; H.C. Mansfield, Taming the prince: the ambivalence of modern executive power, New York, Free Press, 1989.

${ }_{57}$ M. Foucault, Sicurezza, territorio, popolazione. Corso al Collège de France 1977-1978, Milano, Feltrinelli, 2005 (2004), p. 182.

${ }^{58}$ Ibidem.
} 
la trattatistica sulla ragion di stato tratteggia una "economia" del governo politico degli uomini che ha come suo obiettivo la conservazione dell'ordine politico, l'accrescimento della potenza dello Stato e, nei limiti del possibile, anche del benessere dei sudditi/cittadini ${ }^{59}$.

In tal modo, la ragion di Stato ridimensiona la centralità generalmente accordata alla sovranità e alle teorie del diritto naturale mostrando quanto la modernità costituisca una costellazione di istanze differenti. Richiamandoci alla necessità interpretare la modernità politica come l'incrocio di percorsi diversi, che rispondono ai problemi che di volta in volta emergevano nei diversi contesti a partire dall'esercizio effettivo dei poteri e da una comune esigenza di razionalizzazione. Contro l'interpretazione meineckiana, si dovrebbe allora argomentare che nella ragion di Stato lo Stato si scopre in quanto risultato di una contingenza razionale del governo, effettiva "peripezia del governo", più che senso e fine ultimo della storia e della politica.

\section{BIBLIOGRAFIA}

Accetto T. (1641), Della dissimulatione honesta, Napoli, Egidio Longo, 1641.

Albergati F. (1627), La republica regia, Bologna, Vittorio Benacci, 1627.

Ammirato S. (1594), Discorsi sopra Cornelio Tacito, Fiorenza, F. Giunti, 1594.

Arienzo A., Borrelli G. (2011), Emergenze democratiche. Ragion di stato, Governance, Gouvernementalité, Napoli, Giannini Editore, 2011.

Baldini A. E. (a cura di, 1995), Aristotelismo politico e ragion di Stato, Atti del Convegno internazionale di Torino, 11-13 febbraio 1993, Firenze, Olschki, 1995.

(a cura di, 1999), La ragion di Stato dopo Meinecke e Croce: dibattito su recenti pubblicazioni, Atti del Seminario internazionale di Torino, 21-22 ottobre 1994, Genova, Name Press, 1999.

Berkowitz D.S. (1975), Reason of State in England and the Petition of Right, 1603-1629, in Staatsräson, a cura di R. Schnur, Berlin, Duncker \& Humblot, 1975, pp. 165-212.

Boccalini T. (1612), Ragguagli di Parnaso. Centuria prima, Venezia, Pietro Farri, 1612.

Bonaventura F. (1623), Della Ragion di Stato et della prudenza politica libri quattro, Urbino, Alessandro Corvini, 1623.

Borrelli G. (1993), Ragion di Stato e Leviatano. Conservazione e scambio alle origini della modernità politica, Bologna, Il Mulino, 1993.

(1994), Ragion di Stato. L'arte italiana della prudenza politica, in Ragion di Stato. L'arte italiana della prudenza politica, catalogo della mostra bibliografica, Napoli, Palazzo Serra di Cassano 4-30 luglio 1994, Napoli, IISF, 1994.

\footnotetext{
${ }^{59}$ Cfr M. Senellart, Les arts de gouverner. Du regimen médieval au concept de gouvernement, Éditions du Seuil, Paris 1995 (tr. it. Le arti di governare, Napoli, Edizioni Scientifiche Italiane, 2014).
} 
(2000), Non far novità. Alle radici della cultura italiana della conservazione politica, Napoli, Bibliopolis, 2000.

(2015), Gli studi di Ragion di Stato negli ultimi due decenni del ventesimo secolo: motivazioni e considerazioni critiche, "Politics. Rivista Italiana di Studi Politici", vol.4, n.2, 2015, pp.1-13.

(2016), Machiavelli e la ragion di Stato: segnare con cura le differenze, in Stato, Nazione, cittadinanza. Studi di pensiero politico in onore di Leonardo La Puma, a cura di R. Bufano, Lecce, Milella, 2016, pp. 51-64.

(2017), Machiavelli, ragion di Stato, polizia cristiana. Genealogie I, Napoli, Cronopio, 2017.

Borrelli G. (a cura di, 1999), Prudenza civile, bene comune, guerra giusta. Percorsi della ragion di Stato tra Seicento e Settecento, Atti del Convegno internazionale, 22-24 maggio 1996, Napoli, ARS, 1999.

Borrelli G., Coccoli L. (a cura di, 2019), Ragion di Stato e Ragioni della Chiesa, Napoli, Bibliopolis, 2019.

Botero G. (1589), Della ragion di stato libri dieci di Giouanni Botero benese, in Venetia, appresso i Gioliti, 1589.

(1596), Della ragion di stato libri dieci di Giouanni Botero benese, in Torino, appresso Gio. Dominico Tarino, 1596.

(1598), Aggiunte alla Ragion di Stato, Venetia, presso Gio. Battista Ciotti, 1598.

(1607), I Capitani, Torino, appresso G. Tarino, 1607.

Campi A., De Luca S. (a cura di, 2014), Il realismo politico. Figure, concetti, prospettive di ricerca, Soveria Mannelli, Rubbettino, 2014.

Canonieri A. (1609), Il perfetto corteggiano, et dell'Uffizio del Prencipe verso il Cortegiano, Romae, per Bartol. Zannetti, 1609.

(1609), Quaestiones ac discursus in duos primos libros Annalium C. Cornelii Taciti, Romae, ap. Barthol. Zanettum, 1609.

(1614), Dell'introduzione alla Politica, alla Ragion di Stato e alla pratica del buon governo, Anversa, appr. Iochino Trognesio, 1614.

Chiaramonti S. (1635), Della Ragione di Stato, Fiorenza, stamp. di Pietro Nesti, 1635.

Continisio C. (2009), Introduzione a G. Botero, La Ragion di Stato, Roma, Donzelli, 2009

De Mattei R. (1979), I1 problema della "Ragion di Stato" nell'età della Controriforma, Milano-Napoli, Ricciardi, 1979.

de' Calderini A. (1597), Discorsi sopra la Ragione di Stato di Giovanni Botero, Milano, P. M. Locarno, 1597.

Della Casa G. (1558), L'Orazione a Carlo V si trova in Rime, et prose, Vinegia, presso Niccolò Bevilacqua, 1558.

Descendre R. (2016), Introduzione a G. Botero, Della Ragion di Stato, Torino, Einaudi, 2016. 
Dini V. (1983), La prudenza da virtù a regola di comportamento: tra ricerca del fondamento ed osservazione empirica, in V. Dini, G. Stabile, Saggezza e prudenza. Studi per la ricostruzione di un'antropologia nella prima età moderna, Napoli, Liguori Editore, 1983, pp. 13-125.

(2000), Il governo della prudenza. Virtù dei privati e disciplina dei custodi, Milano, FrancoAngeli, 2000.

Dreitzel H. (1970), Protestantischer Aristotelismus und absolutistischer Staat, Wiesbaden, 1970.

du Plessis A. (1688), cardinal duc de Richelieu, Testament Politique, Paris, 1688.

Firpo L. (1976), La ragion di Stato. Appunti e testi, Torino, Bottega d'Erasmo, 1976.

Foucault M. (1997), Il faut défendre la société: cours au Collège de France, 1976, éd. M. Bertani, A. Fontana, Paris, Seuil-Gallimard, 1997.

(2004), Sécurité, territoire, population. Cours au Collège de France, 1977-1978, sous la dir. de F. Ewald et A. Fontana, par M. Senellart, Paris, Seuil-Gallimard, 2004 (tr. it. Sicurezza, territorio, popolazione. Corso al Collège de France 1977-1978, Milano, Feltrinelli, 2005)

Frachetta G. (1592), L'idea del libro de' governi di Stato et di Guerra, Venezia, app. Damian Zenaro, 1592.

(1597), Il Prencipe, Roma, Niccolò Muzio, 1597.

(1617), Il seminario de' governi di Stato, et di guerra, Venezia, per Evangelista

Deuchino, 1617; G.

Frezza F. (1617), Discorsi politici et morali, Napoli, T. Longo, 1617.

Friedrich C.J. (1957), Constitutional Reason of State: the Survival of the Constitutional Order, Providence (R.I.), Brown University Press, 1957.

Guicciardini F. (1521-1526), Del reggimento di Firenze, in Opere, a cura di Roberto Palmarocchi, Milano, Rizzoli, 1941-1942, vol. II.

Harrington J. (1977), A System of Politics, in The Political Works of James Harrington, ed. With an Introduction by J.G.A. Pocock, Cambridge, CUP, 1977, pp.833-854.

Hont I. (2005), Jealousy of trade. International Competition and the Nation-state in Historical Perspective, Cambridge Mass, Belknap Press, 2005.

Jacarandá R. (2016), Os pressupostos filosóficos do maquiavelismo e o surgimento da via moderna em política, "Veritas", 61, 1, 2016, pp.130-153.

Lazzeri C., Reynié D. (1992), La raison d'état: politique et rationalité, Paris, PUF, 1992.

Malvezzi V. (1622), Discorsi sopra Cornelio Tacito, presso Marco Ginammi, Venetia, 1622. (1629), Il Romulo, presso Clemente Ferroni, Bologna, 1629.

(1632), Il Tarquinio Superbo, presso Clemente Ferroni, Bologna, 1632.

(1634), Davide Perseguitato, per Giacomo Monti, Bologna, 1634.

(1635), Il ritratto del privato politico christiano, per Filippo Ghisolfi, Milano, 1635. 
(1648), L'Alcibiade e Il Coriolano, entrambi presso Dozza, Bologna, 1648.

(1989), Taming the prince: the ambivalence of modern executive power, New York, Free Press, 1989.

Maravall J.A. (1972), Estado moderno y mentalidad social. Siglos XV a XVII, 2 voll., Madrid, Revista de Occidente, 1972.

Mazarino G. (1684), Breviarium politicorum secundum rubicas mazarinicas, Coloniae Agrippinae, Typis Iannis Sellica, 1684.

Meinecke F. (1925), Die Idee der Staatsräson in der neuren Geschichte, München und Berlin, Druck und Verlag von R. Oldenbourg, 1925; tr. it. L'idea della ragion di stato nella storia moderna, trad. it. D. Scolari, Sansoni, Firenze 1970.

Mendle M. (1995), Henry Parker and the English Civil War. The political thought of the public's "privado”, Cambridge, CUP, 1995.

Mosse G.L. (1950), The Struggle for Soveraignty in England, East Lansing, Michigan State College Press, 1950.

(1957), The Holy Pretence. A Study in Christianity and Reason of State from William Perkins to John Wintrop, Oxford, Basil, 1957.

Oestreich G. (1989), Filosofia e costituzione dello Stato moderno, Napoli, Bibliopolis, 1989.

Palazzo G.A. (1606), Discorsi del governo e della ragion vera di Stato, Venezia, presso Gio. Antonio e Giacomo de' Franceschi, 1606, (pr. ed. Del governo e della ragion vera di Stato, Napoli, per G.B. Sottile, 1604).

Portinaro P.P. (1999), Il realismo politico, Roma-Bari, Laterza, 1999.

Post G. (1989), Ratio Publicae Utilitatis, Ratio Status and "Reason of State", 1100-1300 in Id., Studies in Medieval Legal Thought: Public Law and the State 1100-1322, Princeton, PUP, 1964, pp.241-309.

Procacci G. (1995), Machiavelli nella cultura europea dell'età moderna, Roma-Bari, Laterza, 1995.

Romano R. (2016), Razão de Estado e Outros Estados da Razão, São Paulo, Editoria Pespectiva, 2014.

Sammarco O. (1628), Delle mutationi de’ regni, Napoli, L. Scoriggio, 1628.

Scattola M. (2016), Politica architectonica. L'aristotelismo politico nel dibattito politico tedesco della prima età moderna, in "Res Publica. Revista de Historia de las Ideas Políticas", 19, 1, 2016, pp.15-33.

Schiera P. (1968), Il cameratismo e l'assolutismo tedesco. Dall'arte di governo alla scienza dello Stato, Milano, Giuffrè, 1968.

(a cura di, 1996), Ragion di Stato e ragioni dello Stato (secoli XV-XVII), Atti del Convegno dell'Istituto per gli studi filosofici e dell'Istituto storico italo-germanico di Trento, 9-10 luglio 1990, Napoli, L’Officina Tipografica, 1996.

(1999), Specchi della politica. Disciplina, melancolia, socialità nell'Occidente moderno, Bologna, Il Mulino, 1999. 
Schito R. (2011), Alla ricerca della sovranità. Fonti e percorsi nella Germania del XVII secolo, Milano, Carocci, 2011.

Schnür R. (hrsg., 1975), Staatsräson. Studien zur geschichte eines politischen Begriffs, Referate des internationalen Kolloquiums über die geschichtliche Rolle des Begriffs der Staatsräson, Tübingen von 2. bis 5. April 1974, Berlin, Verlag Duncker \& Humblot, 1975.

Sebastianelli P. (2017), Homines oeconomici. Per una storia delle arti di governo in età moderna, Roma, Aracne, 2017.

Senellart M. (1989), Machiavélisme et raison d'État, Paris, PUF, 1989.

(1995), Les arts de gouverner. Du regimen médieval au concept de gouvernement, Éditions du Seuil, Paris 1995 (tr. it. Le arti di governare, Napoli, Edizioni Scientifiche Italiane, 2014).

Settala L. (1627), Della Ragion di Stato libri sette, Milano, G.B. Bidelli, 1627.

Stolleis M. (1990), Staat und Staaträson in der frühen Neuzeit. Studien zur Geschichte des öfentlichen Rechts, Suhrkamp, Franckfurt 1990 (tr. it a cura di G. Borrelli, Stato e ragion di stato nella prima età moderna, Bologna, il Mulino, 1998).

Taranto D. (1992), Studi sulla protostoria del concetto di interesse. Da Commynes a Nicole (1524-1675), Napoli, Liguori, 1992.

(1996), Ragion di Stato e ragione di interesse, in P. Schiera (a cura di), Ragion di Stato e ragioni dello Stato, Napoli, L’Officina Tipografica, 1996, pp.189-245.

(2003), Le virtù della politica. Civismo e prudenza tra Machiavelli e gli Antichi, Napoli, Bibliopolis, 2003.

Thuau É. (1966), Raison d'État et pensée politique à l'époque de Richelieu, Paris, Institut français d'Athènes, 1966.

Tuck R. (1993), Philosophy and government, 1572-1651, Cambridge, CUP, 1993.

Viroli M. (1993), Dalla politica alla ragion di Stato. La scienza del governo tra XIII e XVII secolo, Roma, Donzelli, 1993.

Wormuth F. D. (1939), The Royal Prerogative. 1603-1649, New York, Cornell University Press, 1939.

Zinano G. (1625), Il Consigliere, ove si mostra con qual'arte e accortezza debba procedere in tutt' i consigli per ben pubblico d'ogni Stato, Venezia, appr. Gio. Guerigli, 1625.

(1625), Il Segretario, Venezia, appr. Gio. Guerigli, 1625.

(1626), Della Ragione de gli Stati libri XII, Venezia, appr. Gio. Guerigli, 1626.

Zuccolo L. (1621), Considerationi Politiche, e Morali sopra cento oracoli di Illustri Personaggi antichi, Venezia, M. Ginami, 1621. 